\title{
On the Features Solitary Wave Solutions for the Double- Structures Model of DNA
}

\author{
H I Abdel-Gawad, M Tantawy* \\ Department of Mathematics, Faculty of Science, Cairo University, Giza, Egypt
}

\begin{abstract}
In this paper, we propose to describe a nonlinear model from the first principle for the double structures DNA in specific static case, with neglect the chain orientation degrees of freedom. This model includes the base pair stretchings and reduced its great complexity for two main interactions. One is the stacking interaction between neighboring bases along the each chain and another is the nonlinear coupling between two adjacent bases in opposite chain. Here both the discrete and continuum model are performed for $(2+1)$-dimensional to study the nonlinear traveling waves solutions (TWS) on the stretching transition for the long DNA molecules. We discuss the dynamics features, such as amplitudes, velocities in nonlinear traveling waves at the base-pair scales.
\end{abstract}

Keywords: double-structures DNA, discrete, continuum, traveling waves solutions (TWS)

\section{INTRODUCTION}

Deoxyribo-Nucleic Acid (DNA) molecule has been recognized constitutes the basis of the genetic information of this key molecule of life [1]. Since the structure of DNA double helix has been discovered by Watson and Crick in 1953 [2]. Many researchers, especially ones in theoretical physics and nonlinear dynamics and also triggered the formulation of several simple models of nonlinear DNA dynamics corresponding to geometric structure, namely, the DNA double-stranded [3-14] or the double helix [15-28]. These models had been developed during the past decade to describe the nonlinear dynamics properties of open base-pairs in DNA, which commonly called denaturation bubbles [29].

In 1964 Robin Holliday has a well-proposed that double structures of DNA a four-stranded junction would be involved as an intermediate to allow reciprocal exchange of genetic information through recombination across two homologous DNA duplexes [30,31].

In this paper, we introduce a description of mathematical formulation model which incorporates elastic membrane (H-bond) with nonzero distance length between double structures at small stochastic two-degrees of freedom into a discrete version as a continuum distribution in a plane. Further, we estimates of the geometrical and dynamical parameters is used to discuss the dynamics features of each double-stranded wave motions.

\section{DNA MODEL}

In this section, we have used a microscopic model for a double-structures of DNA to describe the dynamics of the openings DNA. From theoretical viewpoints, we ought to take three assumptions (i) we neglect the helical structure of DNA. So that, we shall take four parallel strands. (ii) We consider the transverse motions of DNA strands. (iii) a homogeneous model, i.e. all bases are in this sequence equal, as are the interactions between bases in opposite sites.

Recently, This model includes the neighboring bases are coupled by a harmonic stacking potential $V_{1}$ represent the stacking interaction between neighboring bases along each strand. The nonlinear coupling between two adjacent bases stretched (as the H-bonds between complementary bases), when the four-stranded opens locally is given by the $V_{2}$. Here, we use a Morse potential as fully the nonlinearities to represent the interaction of the bases in a pair [4].

Hence, the model includes four degrees of freedom, $u_{i}, v_{i}$ and $i=n, m$ for the transverse displacements of the four-stranded respectively. Therefore, the equilibrium the displacement of the bases from their equilibrium positions along the direction of $\mathrm{H}$-bonds are 


$$
\left.u_{n, m}=\left(u_{i}+v_{j}\right) /(\sqrt{(}(2)), v_{n, m}=\left(u_{i}-v_{j}\right) /(\sqrt{(}(2)]\right) i, j=n, m, i \neq j[3,11,12] .
$$

The Hamiltonian of such a model of the DNA can be written as:

$H=T+V$,

where the kinetic energy of the elastic strands is

$T=\sum_{n, m=1}^{N}(M) /(2)\left[u_{n, m}^{2}+v_{n, m}^{2}\right]$,

and the potential energy is

$$
\begin{aligned}
& V=V_{1}+V_{2}, \quad V_{1}=(1) /(2)\left[\kappa_{1}\left(\left(u_{n+1, m}-u_{n, m}\right)^{2}+\left(v_{n+1, m}-v_{n, m}\right)^{2}\right)+\kappa_{2}(\right. \\
& \left.\left.\left(u_{n, m+1}-u_{n, m}\right)^{2}+\left(v_{n, m+1}-v_{n, m}\right)^{2}\right)\right], \quad V_{2}=(1) /(2)\left[D_{1} \sum_{n, m=1}^{N}\left(e^{-a, u}{ }_{n, m}-1\right)^{2}+D_{2} \sum_{n=1} N(\right. \\
& \left.\left.\left.e^{-\alpha(v), v_{n, m}-u, m}+h\right)-1\right)^{2}\right]
\end{aligned}
$$

Where $\kappa_{i}, \alpha_{i}^{-1}$ and $D_{i}$ are are an elastic constant, width and depth for each duple-stranded, $i=n, m$ respectively. While $h$ is the distance between tow structures from the center of bases and $M$ is the mass of each nucleotide (taken to be the same for each base).

Then the discrete equations of motion are

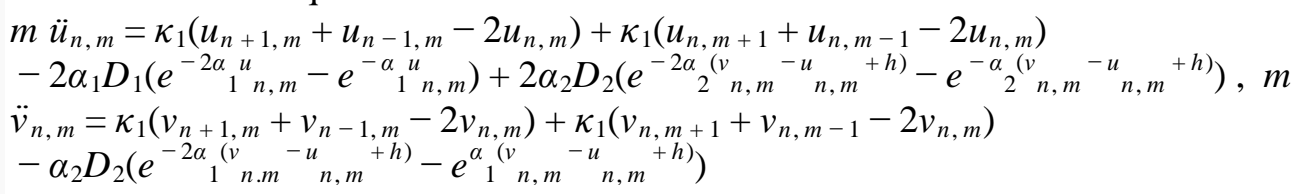

Here, we construct the continuum DNA model. As the distance between two successive bases along the axis of the two chain $\left(\delta \simeq 3.44 A^{\circ}\right)$. Assumptions of the Taylor expansion, we have

$$
\begin{aligned}
& u_{n \pm 1, m}=u_{n, m} \pm \delta\left(\partial u_{n . m}\right) /\left(\partial_{n}\right)+\left(\delta^{2}\right) /(2)\left(\partial^{2} u_{n . m}\right) /\left(\partial_{n}{ }^{2}\right) \pm\left(\delta^{3}\right) /(6)\left(\partial^{3} u_{n . m}\right) /\left(\partial_{n}{ }^{3}\right)+\cdots, \quad u_{n, m \pm 1}=u_{n, m} \pm \delta \\
& \left(\partial u_{n . m}\right) /\left(\partial_{m}\right)+\left(\delta^{2}\right) /(2)\left(\partial^{2} u_{n . m}\right) /\left(\partial_{m}{ }^{2}\right) \pm\left(\delta^{3}\right) /(6)\left(\partial^{3} u_{n . m}\right) /\left(\partial_{m}{ }^{3}\right)+\cdots, \quad v_{n \pm 1, m}=v_{n, m} \pm \delta \\
& \left(\partial v_{n . m}\right) /\left(\partial_{n}\right)+\left(\delta^{2}\right) /(2)\left(\partial^{2} v_{n . m}\right) /\left(\partial_{n}{ }^{2}\right) \pm\left(\delta^{3}\right) /(6)\left(\partial^{3} v_{n . m}\right) /\left(\partial_{n}{ }^{3}\right)+\cdots, \quad v_{n, m \pm 1}=v_{n, m} \pm \delta \\
& \left(\partial v_{n . m}\right) /\left(\partial_{m}\right)+\left(\delta^{2}\right) /(2)\left(\partial^{2} v_{n . m}\right) /\left(\partial_{m}{ }^{2}\right) \pm\left(\delta^{3}\right) /(6)\left(\partial^{3} v_{n . m}\right) /\left(\partial_{m}{ }^{3}\right)+\cdots
\end{aligned}
$$

This suggests that to consider the sequence of bases as a continuum distribution in a plane. Thus we write $u_{i}(t) \simeq u(\delta i, t)$ and $v_{i}(t) \simeq u(\delta j, t), i=n, m$ with $\delta n=x$ and $\delta m=y$. Therefore $u_{n, m}=u(x, y, t)$ and $v_{n, m}=v(x, y, t)[32]$.

By neglecting the terms of $O\left(\delta^{3}\right)$ and higher, so that the equation (4) becomes

$$
\begin{aligned}
& u_{t t}=\kappa_{1} u_{x x}+\kappa_{2} u_{y y}-D_{1}\left(\alpha_{1} u-\left(3 \alpha_{1}\right) /(2) u^{2}+\left(7 \alpha_{1}{ }^{3}\right) /(6) u^{3}\right) \\
& \quad+D_{2}\left(\alpha_{2}(v-u+h)-\left(3 \alpha_{2}{ }^{2}\right) /(2)(v-u+h)^{2}+\left(7 \alpha_{2}{ }^{3}\right) /(6)(v-u+h)^{3}\right), \quad v_{t t}=\kappa_{1} v_{x x}+\kappa_{2} v_{y y}-D_{2}( \\
& \left.\alpha_{2}(v-u+h)-\left(3 \alpha_{2}{ }^{2}\right) /(2)(v-u+h)^{2}+\left(7 \alpha_{2}{ }^{3}\right) /(6)(v-u+h)^{3}\right), .
\end{aligned}
$$

where $\kappa_{1}=\left(\delta^{2} \kappa_{1}\right) /(M), \kappa_{2}=\left(\delta^{2} \kappa_{2}\right) /(M), D_{1}=\left(\alpha_{1} D_{1}\right) /(M)$ and $D_{2}=\left(\alpha_{2} D_{2}\right) /(M)$.

\section{SOLUTIONS OF EQUATION (6)}

The traveling wave solutions TWS of equation (6), are obtained by taking $u(x, y, t)=u(\xi)$, $v(x, y, t)=v(\xi)$ and $\xi=\eta_{1} x+\eta_{2} y+\omega t$. Where $\eta_{1}{ }^{-1}$ and $\eta_{2}{ }^{-1}$ designate the characteristic wavelengths and $\omega$ is the and frequency. Thus, the equation (6) reduce to

$$
\begin{aligned}
& \sigma u^{\prime \prime}+D_{1}\left(\alpha_{1} u-\left(3 \alpha_{1}{ }^{2}\right) /(2) u^{2}+\left(7 \alpha_{1}^{3}\right) /(6) u^{3}\right)-D_{2}\left(\alpha_{2}(v-u+h)\right. \\
& \left.-\left(3 \alpha_{2}{ }^{2}\right) /(2)(v-u+h)^{2}+\left(7 \alpha_{2}^{3}\right) /(6)(v-u+h)^{3}\right)=0, \sigma v^{\prime \prime}+-2 D_{2}( \\
& \left.\alpha_{2}(v-u+h)-(3) /(2) \alpha_{2}{ }^{2}(v-u+h)^{2}+(7) /(6) \alpha_{2}{ }^{3}(v-u+h)^{3}\right)=0,
\end{aligned}
$$

where $\sigma=\left(\omega^{2}-\kappa_{1} \eta_{1}^{2}-\kappa_{2} \eta_{2}{ }^{2}\right)$. By introducing the transformation $v(\xi)=a u(\xi)+b(a$ and $b$ are constants), the equation (7) gives 
$\sigma u^{\prime \prime}+D_{1}\left(\alpha_{1} u-\left(3 \alpha_{1}^{2}\right) /(2) u^{2}+\left(7 \alpha_{1}^{3}\right) /(6) u^{3}\right)+A_{0}=0, \quad \sigma=(1-a) \sigma, A_{0}=-(1) /(3) \alpha_{2}{ }^{2} D_{2}\left(\alpha_{2}(b+h)^{2}\right.$ $\left.\left(7 \alpha_{2}(b+h)-9\right)-6 b+6 h\right)$,

by replacing $u(\xi)=w(\xi)+(3 /) /\left(7 \alpha_{1}\right)$, into equation (8), we have

$\sigma w^{\prime \prime}+\lambda w+\mu w^{3}+L_{0}=0, \lambda=\alpha_{1}\left(\alpha_{1}-(9) /(7)\right), \mu=(7) /(6) \alpha_{1}{ }^{3}$, $L_{0}=\left(7 \alpha_{1}^{3} A_{0}\right) /(6)+\left(3 \alpha_{1}\right) /(7)-(18) /(49), a \neq 1$.

In this work, we have to find the traveling wave solutions TWS of equation (9) by using the unified method UM [33]. The outline of this method are introduced as follows:

(i) Polynomial function solutions

(ii)Rational function solutions.

Here, we confine ourselves to find rational function solutions.

\section{Rational Solutions}

In this case, the solution is constructed by the bilinear transformation form in an auxiliary function that satisfies an auxiliary equation;

$w(\xi)=\sum_{i=0}{ }^{n} a_{i} \varphi^{i}(\xi) / \sum_{i=0}{ }^{r} q_{i} \varphi^{i}(\xi), \quad \varphi^{\prime}(\xi)=\sum_{j=0}{ }^{k p} c_{j} \varphi^{j}(\xi), p=1,2$

Where $a_{i}, q_{i}$ are unknown parameters.

It is worthy to mention that the balance condition in this case be obtained as in the case of polynomial solutions but $n$ is replaced by $n-r$. Here again, the condition for the existence of the solutions of the equation (9) is determined from the consistency equation. Indeed, when $k=1$ in the solution of the second equation in (10) was suggested to describe "a jet stream" or (wave pattern). Thus the first equation in (9) describes nonlinear interactions (NLI) of the wave pattern. Further, we mention that, when $p=1$, the solution of the auxiliary equation gives rise to (explicit or implicit) solutions in elementary functions. While when $p=2$, they give rise to explicit solutions in soliton and Jacobielliptic (or chirped) waves. By noticing that as the equation (9) is invariant under the transformations $(x, y, t) \rightarrow(-x,-y,-t)$ then periodic and elliptic wave solutions exist. That is the case $p=2$ holds here.

The steps of computations are carried in what it follows:

When substituting from equation (10) into the equation (9), we get the principle equations and the following steps are done.

1. Solve the principle equations.

2. Solve the auxiliary equations.

3. Find the exact solution.

4. Check that the solutions obtained are satisfies the equation (9)

(i) Soliton Wave

To find the soliton wave of the equation (9) by using the rational function solution when $(k=$, $p=2$ ), we assume that

$w(\xi)=\left(a_{1} \varphi(\xi)+a_{0}\right) /\left(q_{1} \varphi(\xi)+q_{0}\right), \quad \varphi^{\prime}(\xi)=\sqrt{ }\left(c_{0}+c_{2} \varphi^{2}(\xi)\right)$,

where equation $(11)_{2}$ is the auxiliary equation.

From equation (11) into equation (9), we get the solution is

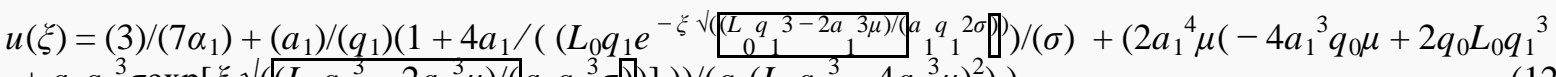

$\left.\left.\left.\left.+a_{1} q_{1}^{3} \sigma \exp \left[\xi \sqrt{ }\left(\left(L_{0} q_{1}^{3}-2 a_{1}^{3} \mu\right) /\left(a_{1} q_{1}^{3} \sigma\right)\right]\right)\right]\right)\right) /\left(q_{0}\left(L_{0} q_{1}{ }^{3}-4 a_{1}^{3} \mu\right)^{2}\right)\right)$ 

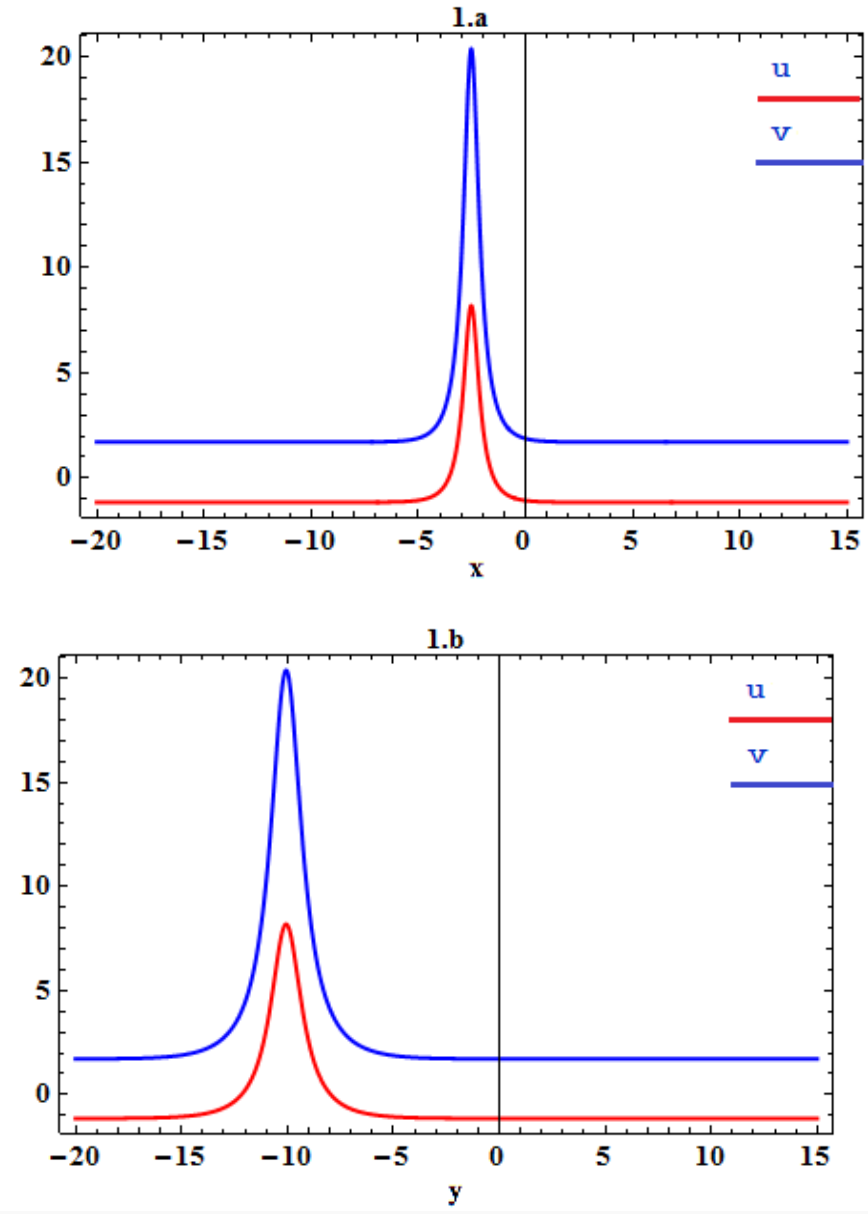

Figs1. (a) and (b) are soliton solutions of the equation (12), against $x$-axis and $y$-axis.

In (a) $q_{0}=1, q_{1}=-2, a_{1}=0.8, \alpha_{1}=\alpha_{2}=0.5, D_{1}=D_{2}=20, h:=0.2, \kappa_{1}=0.7, \kappa_{2}=0.4, \eta_{1}=1.2$, $\eta_{2}=0.6, \omega=0.8, a=2, b=4, y=5$ and $t=0.5$. In (b) at the same values of the parameters in (a), but $x=5$.

Fig. 1(a) and 2(b), representative profiles of the solitonic waves solutions the corresponding to the first structure (red color) and the second structure (blue color) for the one principal direction, say $\mathrm{x}$ direction and weak effects in the transversal direction, say y-direction. These show the double structures waves traveling with the same behavior along the region except the first is weekly than second in the amplitude, width and less steepen.

\section{(ii) Periodic Wave}

We can also find the periodic solution in the second of equation (9) takes

$\varphi^{\prime}(\xi)=\sqrt{ }\left(c_{0}-c_{2} \varphi^{2}(\xi)\right)$

from equation (13) into the equation (9), we get

$\left.\left.u(\xi)=\left[-14 \sqrt{ }(2) a_{1}^{3 / 2} \alpha_{1} q_{1}{ }^{3} \sqrt{ }(\sigma) \sqrt{ }\left(\left(2 a_{1}^{3} \mu\right) /\left(q_{1}{ }^{2}\right)\right]-L_{0} q_{1}\right) \sqrt{\left(\left(L_{0} q_{0}{ }^{2}\left(2 a_{1}{ }^{3} \mu-L_{0} q_{1}{ }^{3}\right)\right) /(\right.} a_{1}{ }^{4} \mu q_{1} \sigma D\right]\right) \quad \sin ((\xi$ $\left.\left.\left.\left.\sqrt{\left(\left(2 a_{1}^{3} \mu\right) /(\right.} q_{1}^{2}\right)-L_{0} q_{1}\right)\right) /\left(\sqrt{ }\left(a_{1}\right) \sqrt{(}(\sigma)\right)\right) L_{0} q_{0} q_{1}^{3}\left(7 a_{1} \alpha_{1}+3 q_{1}\right)(-\cos ((2 \xi$

$\left.\left.\left.\left.\left.\left.\sqrt{\left(\left(2 a_{1}{ }^{3} \mu\right) /(\right.} q_{1}^{2}\right)-L_{0} q_{1}\right)\right) /\left(\sqrt{ }\left(a_{1}\right) \sqrt{ }(\sigma)\right)\right)\right)+B_{0}\right] / 14 \alpha_{1} q_{0} q_{1}\left(L_{0} q_{1}{ }^{3} \sin ^{2}((\xi\right.$ $\left.\left.\left.\left.\left.\sqrt{\left(\left(2 a_{1}^{2} \mu\right) /(\right.} q_{1}^{2}\right)-\left(L_{0} q_{1}\right) /\left(a_{1}\right]\right]\right) /(\sqrt{ }(\sigma \sigma])\right)-2 a_{1}^{3} \mu\right)$,

$B_{0}=q_{0}\left(28 \alpha_{1} a_{1}{ }^{4} \mu-21 \alpha_{1} a_{1} L_{0} q_{1}{ }^{3}-12 a_{1}{ }^{3} \mu q_{1}+3 L_{0} q_{1}{ }^{4}\right)$.

\section{(iii)Elliptic and chirped waves}

To find the elliptic wave solution, the auxiliary equation is taken 
$\left(\varphi^{\prime}(\xi)\right)^{2}=\sum_{i=0}^{j=4} c_{i} \varphi^{j}(\xi)$.

We mention that in the solution obtained $a_{i}$ and $q_{i} i=0,1$ are given in $c_{j}$, where $c_{j}, j=0, \cdots, 4$ are arbitrary parameters.

As there are classified as varieties solutions of the equation (15), we have to take some particular forms for $c_{j}[34,35]$.

For example, we takes

$c_{0}=1-m^{2}, c_{2}=2 m^{2}-1, c_{4}=-m^{2}, \quad c_{1}=c_{3}=0, \varphi(\xi)=\mathbf{c n}\left(\xi, m^{2}\right)$,

thus the solution is

$u(x, t)=\Gamma\left(\left(\Lambda_{0}+\Lambda_{1} \mathbf{c n}\left(\xi, m^{2}\right)\right) /\left(2 m \mathbf{c n}\left(\xi, m^{2}\right)+\sqrt{(2) \sqrt{(}\left(\left(2 m^{2}-1[)\right)\right.}\right)\right)$,

where

$\Lambda_{0}=\sqrt{ }(2)\left(7 \alpha_{1} L_{0}\left(1-2 m^{2}\right) \sqrt{ }(\sigma)+2 \sqrt{ }\left(2 m^{2}-1\right) \sqrt{ }\left(\left[2 m^{2}-1\right] \sigma\right)\left(\lambda+\left(2 m^{2}-1\right) \sigma\right)\right)$,

$\Lambda_{1}=2\left(7 \alpha_{1} L_{0} m \sqrt{ }\left(\left[2 m^{2}-1\right] \sigma\right)+2 \sqrt{ }\left(m^{2}\right) \sqrt{ }\left(2 m^{2}-1\right) \sqrt{ }(\sigma)\left(\lambda+2 m^{2} \sigma-\sigma\right)\right)$,

$\Gamma=(3) /\left(14 \alpha_{1} \sqrt{(} \sigma\left(2 m^{2}-1\right)\left[\left(\lambda+\left(2 m^{2}-1\right][)\right]\right)\right.$,

By bearing in mention, the elliptic (chirped) wave solution is considered particular case of $m$ namely $(m=0.999)[36]$.
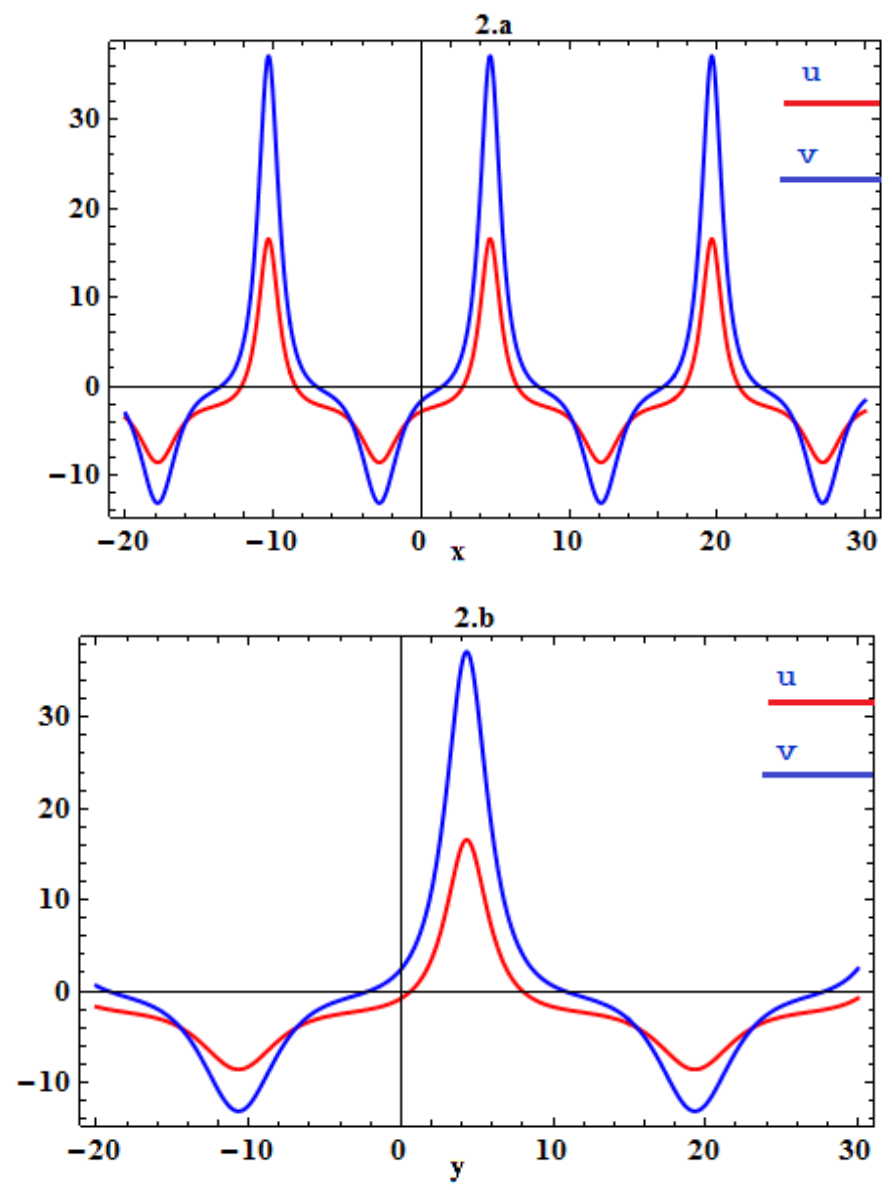

Figs2. (a) and (b) are solutions of the equation (17), at the same caption in figs. $1(a)$ and $1(b)$ respectively and $q_{0}=1, q_{1}=2$ and $m=0.999$.

These figures are the localized elliptic (chirped) waves are generated for the different structure in the $x$-direction and weakly $y$-direction. 


\section{Conclusion}

We present an exact TWS in double structures model of DNA. The UM has been obtained the several nonlinear TWS and the characters of these waves are investigated in detail through defining some values of dependent parameters. Especially, amplitude, velocities and steepen stage pulse with compression is achieved through a continuum distribution in a plane.

\section{REFERENCES}

[1] Alberts B., Johnson A., Lewis J., Raff M., Roberts K., Walter P. , Molecular Biology of the Cell, Gerland Science, New York, 2002.

[2] Watson J.D., Crick F.H.C., Molecular structure of nucleic acids, Nature 171, 737 (1953).

[3] Peyrard M. and Bishop A.R., Statistical mechanics of a nonlinear model for DNA denaturation, Phys. Rev. Lett. 62(23) 2755 (1989); Dauxois T., Peyrard M. and Bishop A.R., Entropy-driven DNA denaturation. Phys. Rev. E 47(1), 44 (1993).

[4] Dauxois T., Peyrard M. and Bishop A.R., Thermodynamics of a nonlinear model for DNA denaturation, Physica D 66 (1993) 35-42; M. Peyrard, T. Dauxois, Hoyet H. and C.R. Willis. Biomolecular dynamics of DNA: statistical mechanics and dynamical models, Physica D 68 (1993) 104-115.

[5] Dauxois T., Peyrard M., Physics of Solitons, Cambridge University Press, 2010.

[6] Yakushevich L.V., Is DNA a nonlinear dynamical system where solitary conformational waves are possible? J. Biosci. 26, 305 (2001).

[7] Singh N. and Singh Y., Statistical mechanics of thermal denaturation of DNA oligomers, Pramana J phys. 61, 345 (2003).

[8] Cuend S. , Sánchez A., On the discrete Peyrard-Bishop model of DNA: stationary solutions and stability, Chaos 16, 023123 (2006).

[9] Sulaiman A., Zen F.P., Alatas H., Handoko L.T., Dynamics of DNA breathing in the PeyrardBishop model with damping and external force, Physica D 241(19), 1640 (2012).

[10] Rapti Z., Stationary solutions for a modified Peyrard-Bishop DNA model with up to thirdneighbor interactions, Eur. Phys. J. E 32, 209 (2010).

[11] Alka W., Goyal A., Nagaraja Kumar C., Nonlinear dynamics of DNA-Riccati generalized solitary wave solutions, Phys. Lett. A 375(3), 480 (2011).

[12] Abdel-Gawad I. H., Tantawy M. and Osman M. S., Dynamic of DNA's possible impact on its damage, Math. Meth. Appl. Sci. 39, 168 (2016).

[13] Alexandrov B. S., Gelev V., Bishop A. R., Usheva A. and Rasmussen K. Ø., DNA breathing dynamics in the presence of a terahertz field, Phys. Lett. A 374, 1214 (2010).

[14] Zdravkovi 'c S. , Satari 'c M.V., DNA dynamics -Experiment proposals, Phys. Lett. A 373, 4453 (2009).

[15] Yakushevich L.V., Nonlinear Physics of DNA, second ed., Wiley, Chichester, 2004, 1998.

[16] Gaeta G., Solitons in Yakushevich-like models of DNA dynamics with improved intrapair potential, J. Nonlinear Math. Phy. 14, 57 (2007); Cadqni M., De Leo R. and Gaeta G., Solitons in a double pendulums chain model, and DNA roto-torsional dynamics, Int. J. Nonlin Mech. 14, 128 (2007) .

[17] Gaeta G., Venier L., Solitary waves in helicoidal models of DNA dynamics, J. Nonlinear Math. Phys. 15, 186 (2008).

[18] Cadoni M., De Leo R., Demelio S., Gaeta G., Twist solitons in complex macromolecules: From DNA to polyethylene, Int. J. Nonlin Mech. 43, 1094 (2009).

[19] Barbi M., Cocco S., Peyrard M., Ruffo S.S., A twist-opening model of DNA", J. Biol. Phys. 24, 97 (1999); Barbi M., Cocco S., Peyrard M., Helicoidal model for DNA opening, Phys. Lett. A 253, 358 (1999).

[20] Agarwal J., Hennig D., Breather solutions of a nonlinear DNA model including a longitudinal degree of freedom. Physica A 323, 519 (2003). 
[21] Qu Z., Kang D.-W., Li Y., Liu W., Liu D.-S., Xie S.-J., Effect of helix angles on polaron motion in poly-DNA, Phys. Lett. A 372, 6013 (2008).

[22] Tabi C. B., Mohamadou A., Kofané T. C., Modulational instability and exact soliton solutions for a twist-opening model of DNA dynamics, Phys. Lett. A 373, 2476 (2009).

[23] Torrellas G., Maciá E., Twist-radial normal mode analysis in double-stranded DNA chains, Phys. Lett. A 376, 3407 (2012).

[24] Huertas M. L., Navarro S., Lopez Martinez M. C., and de la Torre J. G., Simulation of the Conformation and Dynamics of a Double-Helical Model for DNA, Biophys J. 73, 3142 (1997).

[25] Behnia S. , Panahi M., Akhshani A., Mobaraki A., Mean Lyapunov exponent approach for the helicoidal Peyrard-Bishop model, Phys. Lett. A 375, 3574 (2011).

[26] S. Zdravkovi'c, M. V. Satari'c. Transverse interaction in DNA molecule, BioSystems 105 (2011) 10-13.

[27] S. Zdravkovi'c. Helicoidal peyrard-bishop model of DNA dynamics, J. Nonlin Math. Phys. 18 (2011) 463-484.

[28] Zdravkovic S., Sataric M.V., Nonlinear DNA dynamics: nonlinearity versus dispersion, Appl. Math. Comput. 225, 401 (2013).

[29] Albuquerque E.L., Fulco U.L., Freire V. N., Caetano E.W.S., Lyra M. L., de Moura F.A.B.F., DNA-based nanobiostructured devices: The role of quasiperiodicity and correlation effects, Phys. Rep., 535, (4), 139 (2014).

[30] Holliday R., A mechanism for gene conversion in fungi, Genet Res 5, 282 (1964).

[31] Shing Ho P. and Carter M., DNA Structure: Alphabet Soup for the Cellular Soul, DNA Repl.Current Adv. 2011, ch. 1, pp. 1-27.

[32] Ambassa B.G., Motto B. F., Zobo E. B., Kofane C. T., Wave solitons in a coupled left-hande d nonlinear transmission line: Effect of the coupling parameter, Chaos Soliton Fract. 91, 400 (2016).

[33] Abdel-Gawad I. H., Towards a Unified Method for Exact Solutions of Evolution Equations. An Application to Reaction Diffusion Equations with Finite Memory Transport, J. Stat. Phys. 147, 506 (2012).

[34] Zhang L. H, Travelling wave solutions for the generalized Zakharov-Kuznetsov equation with higher-order nonlinear terms, Appl. Math. Comp . 208, 144 (2009).

[35] Fu Z. and Shikuo L., On Some Classes of Breather Lattice Solutions to the sinh-Gordon Equation, Z. Naturforsch. 62, 555 (2007).

[36] Dai C., Wang Y., Yan C., Chirped and chirp-free self-similar cnoidal and solitary wave solutions of the cubic-quintic nonlinear Schrödinger equation with distributed coefficients, Optics Commun. 283, 1489 (2010). 